\title{
PRDM16 is associated with evasion of apoptosis by prostatic cancer cells according to RNA interference screening
}

\author{
SHAOXING ZHU ${ }^{1}$, YIPENG XU ${ }^{1}$, MEI SONG ${ }^{2}$, GUIPING CHEN $^{1}$, HUA WANG $^{1}$, \\ YANG ZHAO $^{1}$, ZONGPING WANG ${ }^{1}$ and FANGYIN LI ${ }^{1}$ \\ Departments of ${ }^{1}$ Urology and ${ }^{2}$ Ultrasonography, Zhejiang Cancer Hospital, Hangzhou, Zhejiang 310022, P.R. China
}

Received May 2, 2015; Accepted June 27, 2016

DOI: $10.3892 / \mathrm{mmr} .2016 .5605$

\begin{abstract}
Histone methylation, which is regulated by histone methyltransferases (HMTs) and histone demethylases (HDMs), has been indicated to be involved in a variety of diseases, particularly in cancer, including androgen-independent prostate cancer (PCa). However, the functions of HMTs and HDTs in cancer have largely remained elusive. The present study, utilized an RNA interference screening using a lentiviral small hairpin (sh)RNA library to systematically elucidate the function of HMTs and HDTs in PCa cell growth and viability. Nine HMTs and HDTs, namely FBXO11, PRDM10, JMJD8, MLL, SETD4, JMJD7, PRMT2, MEN1 and PRDM16, were identified to affect DU145 cell viability, as indicated by an MTS assay subsequent to knockdown of the specific genes using shRNA pools. Furthermore, flow cytometric analysis and western blot analysis of apoptosis-associated proteins indicated that PRDM16 has an anti-apoptotic role in $\mathrm{PCa}$ cells. In addition, the spliced form, sPRDM16/MEL1S, was detected to be overexpressed in PCa cell lines. In conclusion, the present study indicated an important oncogenic role of sPRDM16/MEL1S in PCa and suggested that PRDM16 may represent a novel therapeutic target.
\end{abstract}

\section{Introduction}

Prostate cancer (PCa) is the most common malignancy in males in western countries and the second leading cause of cancer-associated mortality (1). Long-range epigenetic activation (2) and long-range epigenetic silencing (3) have been discovered in the genome of PCa cells, which indicated there were structural re-arrangements in PCa. Post-translational

Correspondence to: Dr Yipeng Xu, Department of Urology, Zhejiang Cancer Hospital, 38 Guangji Road, Hangzhou, Zhejiang 310022, P.R. China

E-mail: xuyp1631@zjcc.org.cn

Key words: histone methyltransferase, histone demethylase, RNA interference screening, apoptosis, PRDM16, MEL1, sPRDM16, MEL1S, FBXO11, PRDM10, JMJD8, MLL, SETD4, JMJD7, PRMT2, MEN1 histone modifications, which represent a classic epigenetic aberration, have been shown to participate in PCa metastasis and androgen-independent transition. A study on castration-resistant $\mathrm{PCa}$ patients revealed that the blockade of histone deacetylases re-sensitized PCa cells to androgen ablation therapy (4). Histone methylation, controlled by histone methyltransferases (HMTs) and histone demethylases (HDMs), was also proved to participate in PCa metastasis and androgen-independent transition. HMTs, such as CAM1 and $\mathrm{EZH} 2$, were found to be over-expressed in $\mathrm{PCa}$, with $\mathrm{EZH} 2$ being regarded as a co-activator of the androgen receptor (AR), showing oncogenic activities in PCa (5). Following the discovery of lysine-specific histone demethylase $1 \mathrm{~A}$ in 2004 (6), histone methylation was initially thought to be irreversible, while its interaction with the AR in vitro and in vivo and its promotion of AR-dependent transcription was demonstrated in 2005 (7). It has been indicated that histone modifications are involved in the regulation of early cell fate decisions, cell differentiation and tissue development, and are also linked with numerous of diseases, particularly cancer (4). HMTs and HDMs participate in a variety of processes which are aberrant in cancers, including DNA damage repair, cell replication and apoptosis $(4,5)$. Altered expression of HMTs and HDMs has been reported to be associated with clinical and pathological outcomes of cancer and to be implicated in the formation of androgen-independent $\mathrm{PCa}$, indicating that these enzymes may represent promising therapeutic targets for $\mathrm{PCa}$.

However, the functions of HMTs and HDTs in cancers as well as the underlying molecular mechanisms have largely remained elusive. The present study performed an RNA interference screening using a lentivirus-directed small hairpin (sh) RNA library targeting all human HMTs and HDTs in order to systematically elucidate the function of HMTs and HDTs in PCa cell growth and viability. PRDM16 was identified to be associated with the evasion of PCa cells from apoptosis, and its spliced form, sPRDM16, was found to be aberrantly expressed in PCa cells.

\section{Materials and methods}

Cell culture, antibodies and shRNA library. The RWPE-1 prostate epithelial (PR) cell line, the BPH-1 benign prostate hyperplasia (BPH) cell line and the DU145, PC-3, PC-3M and 
LNCaP PCa cell lines were purchased from the American Type Culture Collection (Manassas, VA, USA) and maintained at $37^{\circ} \mathrm{C}$ in a humidified atmosphere containing 5\% $\mathrm{CO}_{2}$. The cell culture media of DU145, PC-3, PC-3M, LNCaP, RWPE1, and BPH cell lines was RPMI-1640 (Gibco; Thermo Fisher Scientific, Inc., Waltham, MA, USA). The fetal bovine serum was purchased by Gibco (Thermo Fisher Scientific, Inc). Antibodies against PRDM16 (cat. no. ab106410; 1:500), B-cell lymphoma 2 (Bcl-2; cat. no. ab182858; 1:1,000), Bcl-2 homologous antagonist killer (Bak; cat. no. ab32371; 1:1,000), cleaved caspase-3 (cat. no. ab13847; 1:500) and glyceraldehyde-3-phosphate dehydrogenase (GAPDH; cat. no. ab181602; 1:5,000) were purchased from Abcam (Cambridge, MA, USA). All the antibodies were polyclonal raised in rabbit. The customized lentiviral-mediated shRNA library targeting 88 HMTs and HDMs was purchased from 3D-HTS (Shanghai, China). Each gene was targeted by two pools of four distinct shRNA species with specificity for different sequences of the target transcript.

RNA interference (RNAi) screening. DU145 cells (were plated in 96-well plates at 3,000 per well and transfected using a virus concentration of MOI $=30$, with the shRNA library for $24 \mathrm{~h}$. An 3-(4,5-dimethylthiazol-2-yl)-5-(3-carbo xymethoxyphenyl)-2-(4-sulfophenyl)-2H-tetrazolium (MTS) assay was then performed as described below. Cells were screened in independent triplicates. Four individual shRNA species which targeted each gene were then assessed in order to validate and narrow down the results of the primary screening. RNAi-mediated gene silencing was then confirmed by reverse-transcription quantitative polymerase chain reaction (RT-qPCR) and western blot analysis.

$R T-q P C R$. DU145 cells were seeded into six-well plates and transfected with shRNA for $96 \mathrm{~h}$. TRIzol was used to extract the total RNA and complementary DNA was generated using a ReverTra Ace qPCR RT kit (Toyobo Co., Ltd., Osaka, Japan). A SYBR Green PCR kit (Takara Bio Inc., Otsu, Japan) was then used to amplify the cDNA by PCR. The thermocycling conditions used were, pre-denaturation at $94^{\circ} \mathrm{C}$ for $5 \mathrm{~min}, 30$ cycles of denaturation at $94^{\circ} \mathrm{C}$ for $30 \mathrm{sec}$, annealing at $56^{\circ} \mathrm{C}$ for $30 \mathrm{sec}$ and extension at $72^{\circ} \mathrm{C}$ for $30 \mathrm{sec}$, final step at $72^{\circ} \mathrm{C}$ for $20 \mathrm{~min}$. The primers were purchased from Dingguo Changsheng Biotechnology, Co., Ltd. (Beijing, China) and the following sequences were used: PRDM16, forward (F) 5'-TTCTCTGGA CGCTTGGTTGA-3' and reverse (R) 5'-GAGGCCCTAGAG GTGGTTGAT-3'; PRDM16-L, F 5'-CAAGGAGGAGGAGAG AGATT-3' and R 5'-CGGTTGGGCTCATACATA-3'; and sPRDM16 F 5'-TGCACACCCAGCAACACC-3'and R 5'-GCT GCGCTAGAGAAAAGCGT-3'. The expression of GAPDH was also evaluated to calculate the relative target gene expression. The $2^{-\Delta \Delta \mathrm{Cq}}$ method was used for quantification (8).

Western blot analysis. Cells cultured in six-well plates were lysed with $300 \mu$ l sodium dodecyl sulfate-polyacrylamide gel electrophoresis (SDS-PAGE) lysis buffer. The bicinchoninic acid protein assay (OriGene Technologies, Inc., Beijing, China) was used to evaluate the protein concentration. A total of $30 \mu \mathrm{g}$ protein of each sample was subjected to $8 \%$ SDS-PAGE (OriGene Technologies, Inc.) and then transferred onto polyvinylidene difluoride membranes. Membranes were then blocked in 5\% non-fat milk in Tris-buffered saline/Tween 20 for $1 \mathrm{~h}$ at $37^{\circ} \mathrm{C}$, followed by incubation with the indicated antibodies for $1 \mathrm{~h}$ at $37^{\circ} \mathrm{C}$. Subsequently, the membranes were incubated with horseradish peroxidase-conjugated secondary antibody (cat. no. ab6721; 1:300), and blots were visualized using enhanced chemiluminescence reagent (OriGene Technologies, Inc.).

Cell proliferation assay. Following transfection of DU145 cells with the shRNA library as described above, the transfection medium in each well was replaced with $100 \mu$ cell culture medium. $20 \mu 1$ One Solution Reagent (Promega Corp., Madison, WI, USA) containing $19 \mu \mathrm{l}$ MTS and $1 \mu \mathrm{l}$ phenazine methosulfate was added into each well, followed by incubation at $37^{\circ} \mathrm{C}$ for $1 \mathrm{~h}$. The optical density value of the supernatant was then measured at $490 \mathrm{~nm}$ using a spectrophotometer.

Flow cytometry. DU145 cells were collected and washed with phosphate-buffered saline (PBS) following $120 \mathrm{~h}$ of transfection. They were centrifuged at $400 \mathrm{x}$ g and washed twice. The cells were seeded at a density of $1.0 \times 10^{6}$ cells $/ \mathrm{ml}$ and 1X Annexin-binding buffer was added. Alexa Fluor 488 Annexin V (OriGene Technologies, Inc., Beijing, China) and $100 \mu \mathrm{g} / \mathrm{ml}$ propidium iodide working solution were added to each $100 \mu \mathrm{l}$ of cell suspension. The cells were incubated at room temperature for $15 \mathrm{~min}$. Next, $400 \mu \mathrm{l} 1 \mathrm{X}$ Annexin-binding buffer was added and mixed gently. The fluorescence emission was measured at $530 \mathrm{~nm}$ using a flow cytometer (FC 500 MCL/MPL, Beckman Coulter, Inc., Brea, CA, USA).

Statistical analysis. SPSS version 13.0 (SPSS, Inc., Chicago, IL, USA) and GraphPad Prism 5 (GraphPad Software Inc., California, USA) were used for the statistical analyses. Z-score and a Student's t-test were performed. $\mathrm{P}<0.05$ was considered to indicate a statistically significant difference between values.

\section{Results}

RNAi screening for HMTs and HDMs associated with PCa cell viability. To identify HMTs and $\mathrm{HDMs}$ implicated in $\mathrm{PCa}$ cell viability, a systematic RNAi-mediated screening assay was performed. DU145 cells were transfected with an array of 176 shRNAs targeting a total of 88 HMTs and HDMs. After $24 \mathrm{~h}$ of transfection, the cell viability in each well was evaluated to assess the effects of each pool of shRNAs on the cell growth. Pools were classified as hits if the absolute value of the Z-score was $>1.96$ compared to the negative control (Fig. 1). A total of nine genes were identified by this screen with a bottom Z-score of -2.217 (PRDM16) and a top Z-score of -3.211 (FBXO11) (Table I).

PRDM16 inhibition decreases DU145 cell viability. The present study next evaluated the effects of PRDM16 inhibition on the viability of DU145 cells and explored its implication in apoptosis. To exclude the possibility that the inhibition of DU145 viability was induced by the knockdown experiments due to off-target effects, each well of DU145 cells was transfected with one of four different shRNA lentiviral vectors. As three of the four lentiviral vectors reduced the cell viability 
Table I. Genes identified to be associated with DU145 cell viability in the RNA interference screening.

\begin{tabular}{lccccccccc}
\hline Gene & FBXO11 & PRDM10 & JMJD8 & MLL & SETD4 & JMJD7 & PRMT2 & MEN1 & PRDM16 \\
\hline Z-score & -3.221 & -2.903 & -2.509 & -2.446 & -2.435 & -2.415 & -2.380 & -2.202 & -2.127 \\
\hline
\end{tabular}

by $>70 \%$ (Fig. 2), the presence of an off-target effect could be excluded. The results revealed that 0034-07D exerted the highest inhibitory effect on DU145 cells; therefore, this shRNA was used in all subsequent experiments. PCR and western blot analyses confirmed a reduction of PRDM16 expression by 0034-07D at the mRNA and protein level.

PRDM16 inhibition upregulates DU145 cell apoptosis. To explore the mechanism by which PRDM16 regulates cell viability, flow cytometric analysis was used to evaluate the apoptotic rate of DU145 cells. The results indicated that inhibition of PRDM16 induced DU145 cell apoptosis (Fig. 3); suggesting that PRDM16 is involved in the evasion of apoptosis of PCa cells, while the study of its effects on cell proliferation exceeded the margin of the present study. To explore the mechanism by which PRDM16 regulates apoptosis, the expression of several apoptotic proteins was evaluated using western blot analysis. The results indicated that the increase of apoptosis after PRDM16 inhibition was parallelled with downregulation of the expression of the anti-apoptotic Bcl-2 and upregulation of the pro-apoptotic Bak and cleaved caspase- 3 .

sPRDM16/MELIS has an oncogenic role in PCa. Next, the present study evaluated the expression of PRDM16/MEL1 and its spliced version, sPRDM16/MEL1S, in the RWPE-1 $\mathrm{PE}$ cell line, the BPH-1 BPH cell line and four prostate cancer cell lines, DU145, PC-3, PC-3M and LNCaP (Fig. 4). RT-qPCR showed that PRDM16/MEL1 levels were decreased in most PCa cell lines, while sPRDM16/MEL1S was enhanced compared with that in the PE cell line. Furthermore, western blot analysis showed that PRDM16/MEL1 was present in each cell line, while sPRDM16/MEL1S was only detected in three out of four PCa cell lines (DU145, PC-3 and LNCaP), which indicated an oncogenic role of sPRDM16/MEL1S in PCa.

\section{Discussion}

To the best of our knowledge, the present study was the first to use a RNAi screening-based approach to systematically assess the effects of all human HMTs and HDTs on PCa cell viability. By using this approach, nine genes associated with PCa cell viability were identified, of which FBOX11, PRMT2, MEN1, MLL, PRDM16 and SETD4 have already been reported to be abnormally expressed in various cancer types (9-14). The PRDM16 gene is rearranged in AML and MDS (13). Approximately $25 \%$ of primary prostate tumors that progress to metastasis have gained this at the MEN1 locus (11). SETD4 was important for breast carcinogenesis and may be a novel molecular target for the diagnosis and treatment of breast carcinoma (14). While JMJD7 and -8 were identified by the screening of the present study, JMJD2 family proteins are well known to be associated with a variety of cancer types, including PCa (15).

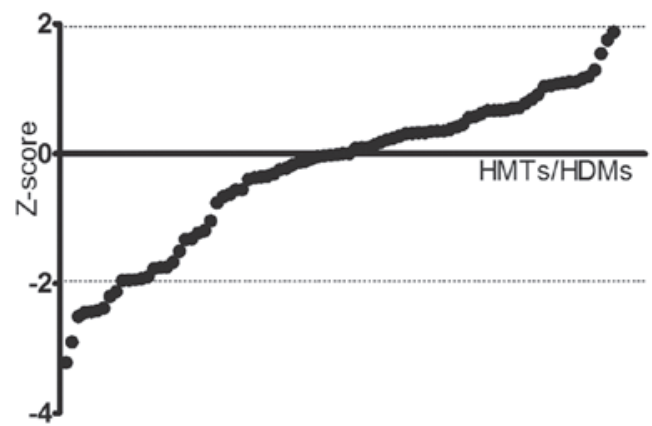

Figure 1. RNA interference screening with a lentiviral shRNA library targeting $88 \mathrm{HMT} / \mathrm{HDM}$ genes. DU145 cells were transfected with a library of lentiviral shRNAs targeting $88 \mathrm{HMT} / \mathrm{HDM}$ genes, with each gene targeted by two pools of four distinct shRNAs specific for a different sequence. Cell viability was evaluated using an MTS assay after $24 \mathrm{~h}$ of transfection. Nine positive genes were obtained in this screen with an absolute value of the Z-score of $>1.96$ compared with the negative control. shRNA, small hairpin RNA; HMT, histone methyltransferase; HDM, histone demethylase.

Among the nine genes identified by the present study, MEN1 has been previously reported to be involved in the tumorigenesis of PCa (11), which indicated high accuracy of the screening performed and suggested that RNAi screening is a powerful tool to identify genes involved in cancer.

Furthermore, the present study revealed that PRDM16 inhibition decreased DU145 cell viability by increasing the apoptotic rate. To explore the underlying mechanisms by which PRDM16 regulates apoptosis, the expression of several apoptotic genes was evaluated using western blot analysis. The results revealed that RNAi of PRDM16 induced apoptosis by reducing the expression of Bcl-2 and by upregulating the expression of Bak. Furthermore, the induction of apoptosis was associated with activation of caspase- 3 by its cleavage.

PRDM16 is a member of the family of PR domain-containing proteins, which consists of 17 members and is characterized by the PR domain with a variable number of $\mathrm{Zn}$-fingers (16). They function as regulators of chromatin function and transcription factors which are involved in the determination of cell fate. PRDM16 serves important functions in adipose tissue differentiation, and has also been described as an oncoprotein in myelodysplastic syndrome, acute myelocytic leukemia, adult T-cell leukemia and gastric carcinoma $(13,17,18)$. sPRDM16, an alternatively spliced form of PRDM16 lacking the PR domain, was described to be aberrantly expressed in AML and gastric carcinoma. sPRDM16, but not PRDM16, was able to prevent TGF- $\beta$-induced growth inhibition in mouse $\mathrm{T}$ cells $(17,18)$. The present study provided the first evidence that SPRDM16 is aberrantly expressed in PCa cell lines (DU145, PC-3 and LNCaP), while its expression could not be detected in the RWPE-1 PE cell line and the BPH-1 BPH cell line. These results suggested that sPRDM16 may have an oncogenic function in PCa and may represent a novel therapeutic target. 


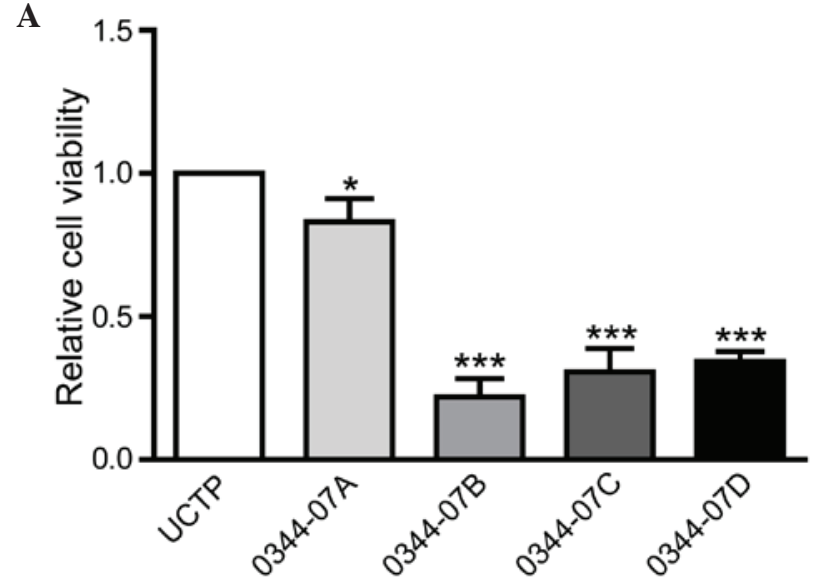

B

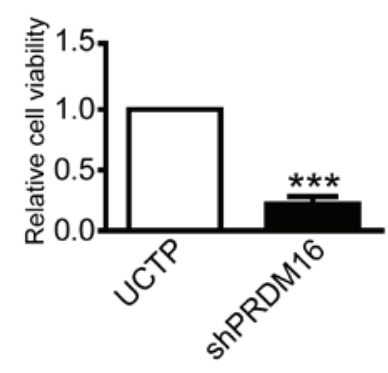

C

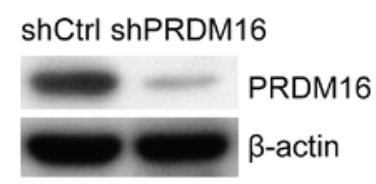

Figure 2. Effects of PRDM16 on cell viability. (A) The PRDM16-2 shRNA pool had been identified to affect cell viability by a preliminary screening. Therefore, the effects of its four individual shRNA species, 0034-07A, 0034-07B, 0034-07C and 0034-07D, on the viability of DU145 cells was assessed. Among them, 0034-07D showed highest inhibitory effect. (B) Polymerase chain reaction analysis confirmed that PRDM16 mRNA expression in DU145 cells was markedly decreased after transfection of 0034-07D. (C) Western blot analysis confirmed that PRDM16 protein expression in DU145 cells was markedly decreased after transfection of 0034-07D. ${ }^{*} \mathrm{P}<0.05 ;{ }^{* * *} \mathrm{P}<0.001$. shRNA, small hairpin RNA; UCTP, control group.

The screening approach of the present study provided nine genes involved in the regulation of PCa cell viability. The detailed results on PRDM16, and previous results on MEN1 (11), which were among these nine genes, indicate that the other seven genes may also have oncogenic roles in $\mathrm{PCa}$, which requires further study. As BOX11, PRMT2, MLL and SETD4 have been previously indicated to be linked with other cancer types, their association with PCa is likely $(8,9,11,13)$. Furthermore, JMJD7 and -8 and PRDM10 are members of the JMJD and PRDM family, respectively indicating similar functions to those of JMJD2 (15) and PRDM16.

It has been previously suggested that histone methylation, controlled by HMTs and HDMs, occurs during PCa metastasis and androgen-independent transition. The present study indicated that nine HMT/HDM genes are involved in the regulation of PCa cell viability, and that the inhibition of PRDM16 decreased DU145-cell viability via enhancement of the apoptotic rate. SPRDM16, an alternatively spliced form of PRDM16 lacking the PR domain, was identified to be overexpressed

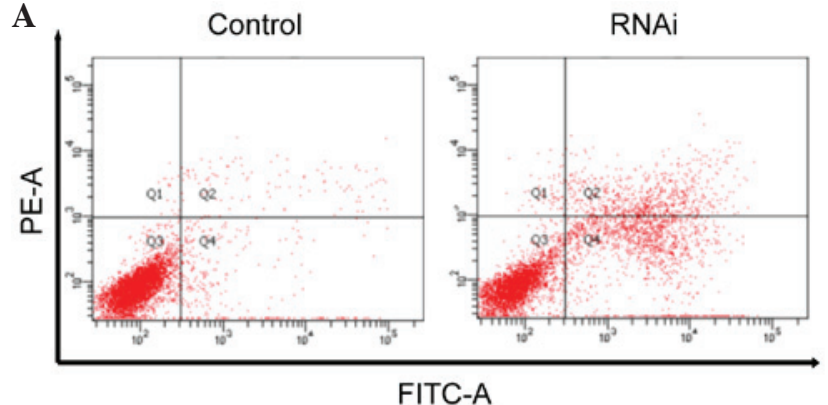

B

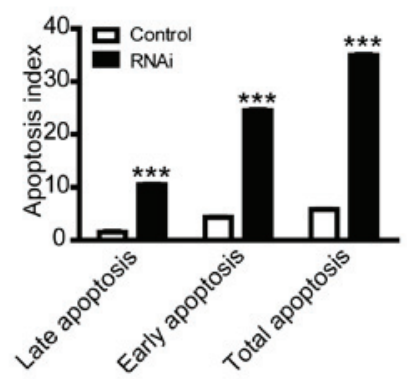

C

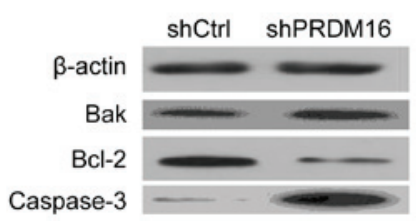

Figure 3. PRDM16 inhibition up-regulates DU145 cells apoptosis. (A and B) Flow cytometric analysis indicated that the apoptotic rate of DU145 cells was markedly increased by PRDM16 inhibition. (C) Western blot analysis indicated that knockdown of PRDM16 resulted in the downregulation of Bcl-2 expression and upregulation of Bak and cleaved caspase- 3 . $^{* * *} \mathrm{P}<0.001 \mathrm{Bcl}-2$, B-cell lymphoma 2; BAK, Bcl-2 homologous antagonist killer; RNAi, RNA interference; shRNA, small hairpin RNA.

$\mathbf{A}$

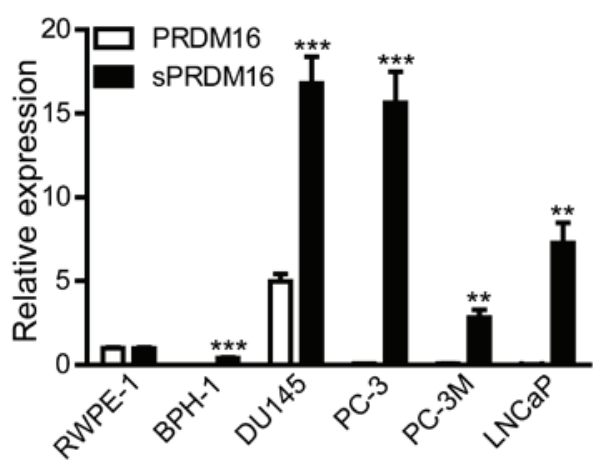

B

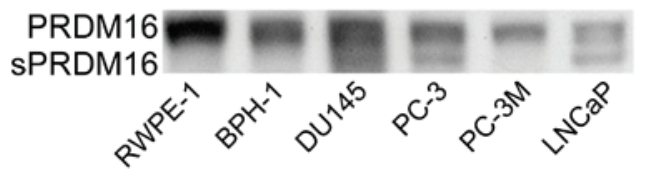

Figure 4. Levels of PRDM16/MEL1 and its spliced form sPRDM16/MEL1S in PE (RWPE-1), BPH (BPH-1) and PCa (DU145, PC-3 and LNCaP) cell lines. (A) Polymerase chain reaction analysis indicated that the levels of PRDM16/MEL1 were downregulated in BPH and most PCa cell lines, while sPRDM16/MEL1S levels were increased compared with those in the PE cell line. (B) Western blot analysis indicated that PRDM16/MEL1 was present in each cell line, while sPRDM16/MEL1S was detected in three of the four PCa cell lines only, which indicated an oncogenic role of sPRDM16/MEL1S in PCa. ${ }^{* *} \mathrm{P}<0.01 ;{ }^{* * *} \mathrm{P}<0.001$. $\mathrm{PCa}$, prostate cancer; $\mathrm{BPH}$, benign prostate hyperplasia; PE, prostate epithelial. 
in three out of four PCa cell lines. These results suggested that sPRDM16 has an oncogenic role in PCa. While MEN1 has been previously reported to be associated with $\mathrm{PCa}$, the likely oncogenic roles of the other seven genes, which have been partly indicated in other types of cancer, remain to be examined by further studies.

\section{Acknowledgements}

The present study was funded by a project supported by the Province-Ministry Incubation Program (no. 2014PYA004), Appropriate Technical Transformation of Zhejiang province (nos. 2013ZHB001 and 2014ZHB001) and the Natural Science Funds Of Zhejiang Province (no. LY16H160034).

\section{References}

1. Siegel RL, Miller KD and Jemal A: Cancer statistics, 2015. CA Cancer J Clin 65: 5-29, 2015.

2. Bert SA, Robinson MD, Strbenac D, Statham AL, Song JZ, Hulf T, Sutherland RL, Coolen MW, Stirzaker C and Clark SJ: Regional activation of the cancer genome by long-range epigenetic remodeling. Cancer Cell 23: 9-22, 2013.

3. Coolen MW, Stirzaker C, Song JZ, Statham AL, Kassir Z, Moreno CS, Young AN, Varma V, Speed TP, Cowley M, et al: Consolidation of the cancer genome into domains of repressive chromatin by long-range epigenetic silencing (LRES) reduces transcriptional plasticity. Nat Cell Biol 12: 235-246, 2010.

4. Crea F, Sun L, Mai A, Chiang YT, Farrar WL, Danesi R and Helgason CD: The emerging role of histone lysine demethylases in prostate cancer. Mol Cancer 11: 52, 2012.

5. Albert $\mathrm{M}$ and Helin K: Histone methyltransferases in cancer. Semin Cell Dev Biol 21: 209-220, 2010.

6. Shi Y, Lan, F, Matson C, et al: Histone Demethylation Mediated by the Nuclear Amine Oxidase Homolog LSD1. Cell 119: 941-953, 2004

7. Metzger E, Wissmann M, Yin N, Müller JM, Schneider R, Peters AH, Günther T, Buettner R and Schüle R: LSD1 demethylates repressive histone marks to promote androgen-receptor-dependent transcription. Nature 437: 436-439, 2005.
8. Livak KJ and Schmittgen TD: Analysis of relative gene expression data using real-time quantitative PCR and the 2(-Delta Delta C(T)) method. Methods 25: 402-408, 2001.

9. Duan S, Cermak L, Pagan JK, Rossi M, Martinengo C, di Celle PF, Chapuy B, Shipp M, Chiarle R and Pagano M: FBXO11 targets BCL6 for degradation and is inactivated in diffuse large B-cell lymphomas. Nature 481: 90-93, 2012.

10. Baldwin RM, Morettin A, Paris G, Goulet I and Côté J: Alternatively spliced protein arginine methyltransferase 1 isoform PRMT1v2 promotes the survival and invasiveness of breast cancer cells. Cell Cycle 11: 4597-4612, 2012.

11. Paris PL, Sridharan S, Hittelman AB, Kobayashi Y, Perner S, Huang G, Simko J, Carroll P, Rubin MA and Collins C: An oncogenic role for the multiple endocrine neoplasia type 1 gene in prostate cancer. Prostate Cancer Prostatic Dis 12: 184-191, 2009.

12. Angelova S, Jordanova M, Spassov B, Shivarov V, Simeonova M, Christov I, Angelova P, Alexandrova K, Stoimenov A, Nikolova V, et al: Amplification of c-MYC and MLL genes as a marker of clonal cell progression in patients with myeloid malignancy and trisomy of chromosomes 8 or 11. Balkan J Med Genet 14: 17-24, 2011.

13. Duhoux FP, Ameye G, Montano-Almendras CP, et al: PRDM16 (1p36) translocations define a distinct entity of myeloid malignancies with poor prognosis but may also occur in lymphoid malignancies. Br J Haematol 156: 76-88, 2012.

14. Faria JA, Corrêa NC, de Andrade C, et al: SET domain-containing protein 4 (SETD4) is a newly identified cytosolic and nuclear lysine methyltransferase involved in breast cancer cell proliferation. J Cancer Sci Ther 5: 58-65, 2013.

15. Berry WL and Janknecht R: KDM4/JMJD2 histone demethylases: Epigenetic regulators in cancer cells. Cancer Res 73: 2936-2942, 2013.

16. Pinheiro I, Margueron R, Shukeir N, Eisold M, Fritzsch C, Richter FM, Mittler G, Genoud C, Goyama S, Kurokawa M, et al: Prdm3 and Prdm16 are H3K9me1 methyltransferases required for mammalian heterochromatin integrity. Cell 150: 948-960, 2012.

17. Yoshida M, Nosaka K, Yasunaga J, Nishikata I, Morishita K and Matsuoka M: Aberrant expression of the MEL1S gene identified in association with hypomethylation in adult T-cell leukemia cells. Blood 103: 2753-2760, 2004.

18. Takahata M, Inoue Y, Tsuda H, Imoto I, Koinuma D, Hayashi M, Ichikura T, Yamori T, Nagasaki K, Yoshida M, et al: SKI and MEL1 cooperate to inhibit transforming growth factor-beta signal in gastric cancer cells. J Biol Chem 284: 3334-3344, 2009. 